\title{
Temporal peculiarities of brittle fracture of rocks and concrete
}

\author{
Y. Petrov, I. Smirnov, A. Evstifeev, N. Selyutina \\ St. Petersburg State University, Universitetsky prospekt, 28, Peterbof, 198504, St. Petersburg (Russian Federation) \\ yp@YP1004.spb.edu; ivansmirnov@math.spbu.ru
}

\begin{abstract}
When we want to compare the strength of two materials, we compare the table values of quasistatic strength. However results of some experiments show that the strength ratio of the materials can change with increase in rate of loading. Such "substitution effect" of material strength at different strain rates is studied in this paper. It is shown that one material can have a lower dynamic strength for a higher static strength compared to the other material. Tests of two different materials, tests of mortar and concrete, and tests of concrete with different water content are considered. It is shown that load-carrying capacity of materials and the substitution effect of material strength in a wide range of loading rates can be predicted by the incubation time criterion.
\end{abstract}

KEYWORDS. Static strength; Dynamic strength; Incubation time; Substitution effect.

\section{INTRODUCTION}

I $\mathrm{n}$ selecting a material of construction an engineer is usually guided by the values of mechanical parameters obtained in quasi-static tests. A construction material is selected on the basis of its ability to withstand a certain stress (as one of the defining parameters). There is a set of test standards governing determination of the ultimate strength of a material under quasi-static tension, compression, bending, etc. However tests under dynamic loading conditions show essential differences of dynamic strength characteristics in comparison with those of quasi-static tests.

These differences relate to the dependence of the mechanical characteristics on the history (duration, intensity, shape of the stress pulse, etc.) and method of loading [1]. A critical value can be considered as a material constant only under quasistatic loading conditions. Under dynamic loading, the critical properties are characterized by very strong instabilities and can vary by several orders of magnitude. As a result, the dynamic system behaviour often appears unpredictable.

Importance of studying rocks and concrete dynamic strength is not in doubt. These materials are highly suitable materials for building structures in the energy sector, for instance solar energy storage water tanks, nuclear containment vessels, etc. However, such civil and military infrastructures (e.g. buildings, barracks, bridges, tunnels, containment structures for hazardous, toxic and inflammable materials, etc) are susceptible to catastrophic failure under intense sudden overloading (e.g. due to blasts, explosions) for which they are not designed. A correct and rational analysis of dynamically loaded structures requires an understanding of the behaviour of the mechanical properties of materials at high strain rates. Therefore a study of the behaviour of rocks and concrete at high rates of deformation and fracture is necessary.

Dependence of rocks on the extraction quarry, as well as numerous admixtures of concrete makes the research of strength characteristics a complex task. Moreover, various heterogeneity and impurity content can lead to various dynamic effects of fracture.

One of such effects is the change of the dominant strength between the two materials. A material, which has a lower strength compared to another material in quasi-static tests, can have greater strength under dynamic loading. 
In this paper we consider such "substitution effect" of strength of two materials in three cases: tests of two different materials; tests of mortar and concrete; tests of concrete under different environmental conditions. As experimental data we used the results of [2-5]. The theoretical analysis is based on the criterion of the incubation time of fracture $[1,6,7]$. This criterion provides correct transition between quasi-static and dynamic loadings. Introduction of the additional measured characteristic of strength (the incubation time) to the already known "quasi-static" parameter of strength (limit stress) allows us to build dependences of maximal fracture stress on a loading (deformation) rate without numerous experiments for any type and character of loading.

\section{THE INCUBATION TIME CRITERION}

7 he criterion of fracture based on the concept of incubation time, proposed in [6-8], makes it possible to predict the unstable behaviour of the dynamic-strength characteristics observed in experiments on the dynamic fracture of solids. The fracture criterion can be written in the following general form:

$$
\frac{1}{\tau} \cdot \int_{t-\tau}^{t}\left(\frac{F\left(t^{\prime}\right)}{F_{c}}\right)^{\alpha} d t^{\prime} \leq 1
$$

Here, $F(t)$ is the intensity of the local force field causing the fracture (or structural transformation) of the medium, $F_{c}$ is the static limit of the local force field, and $\tau$ is the incubation time associated with the dynamics of the relaxation processes preceding the fracture. It actually characterizes the strain (stress) rate sensitivity of the material. The fracture time $t^{*}$ is defined as the time at which equality sign is reached in Eq. (1). The parameter $\alpha$ characterizes the sensitivity of the material to the intensity (amplitude) of the force field causing the fracture (or structural transformation). Often, $a=1$ gives a good agreement with test data.

One of the possible means of interpreting and determining the parameter $\tau$ is proposed here on the example of the mechanical rupture of a material. Let us assume that a standard test specimen made of the material in question is subjected to tension and is broken into two parts under a stress $P$ arising at a certain time $t=0: F(t)=P H(t)$, where $H(t)$ is the Heaviside step function. In the case of quasi-brittle fracture, the material would unload, and the local stress at the break point would decrease rapidly (but not instantaneously) from $P$ to 0 . In this case, a corresponding unloading wave is generated which propagates over the sample and can be detected by standard (e.g., inter-ferometry) methods. The stress variation at the break point can be conditionally represented by the relation $\sigma(t)=P-P f(t)$, where $f(t)$ varies from 0 to 1 (Fig. 1) within a certain time interval $T$.

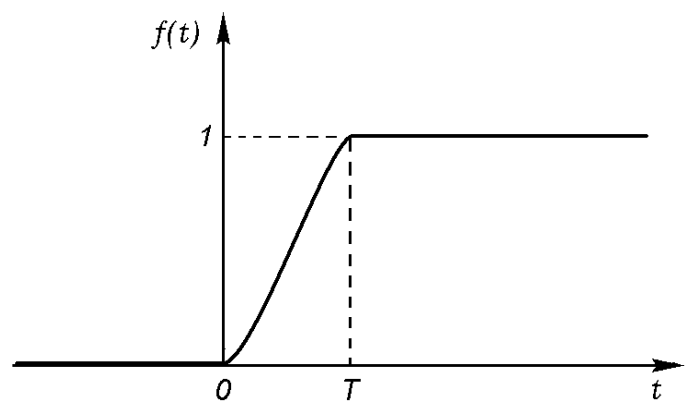

Figure 1: Schematic of fracture kinetics at the place of rupture.

The case $f(t)=H(t)$ corresponds to the classical strength theory. In other words, according to the classical approach, rupture occurs instantaneously $(T=0)$. In practice, the rupture of a material (sample) is a process in time, and the function $f(t)$ describes the micro- scale level kinetics of the transition from a conditionally defect-free state $(f(0)=0)$ to a completely broken state at the given point $f\left(t^{*}\right)=1$ that can be associated with the macro-fracture event. On the other hand, application of the fracture criterion (1) to macro-level situation $(F(t)=P H(t))$, gives the time to fracture $t^{*}=T=\tau$ at $P=F_{c}$.

In other words, the incubation time introduced above is equal to the duration of the fracture process after the stress in the material has reached the static strength on the given scale level [9]. This duration can be measured experimentally by statically fracturing the samples and controlling the rupture process by different possible methods, e.g., by measuring the time of 
the increase in pressure at the unloading wave front based on the recorded velocity profile of points (by interferometry) on the sample boundary. Below, we apply the general fracture criterion Eq. (1) to three problems.

\section{Calculation PROCEDURe}

$\mathrm{L}$

et us consider application of the criterion (1) for calculation of carrying capacity of a material for different strain rate. Note that we understand the carrying capacity as the maximum stress that the material can withstand without fracture. However according to the criterion of incubatory time, dynamic strength is characterized by the incubatory time, but not the maximum stress.

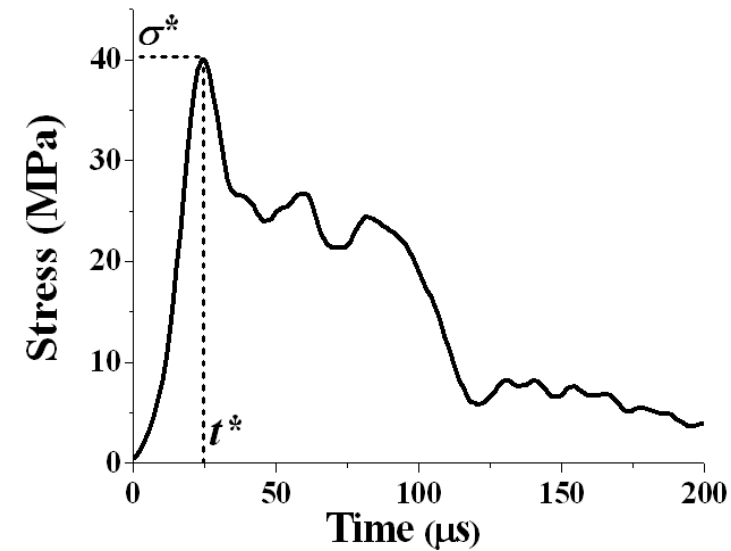

(a)

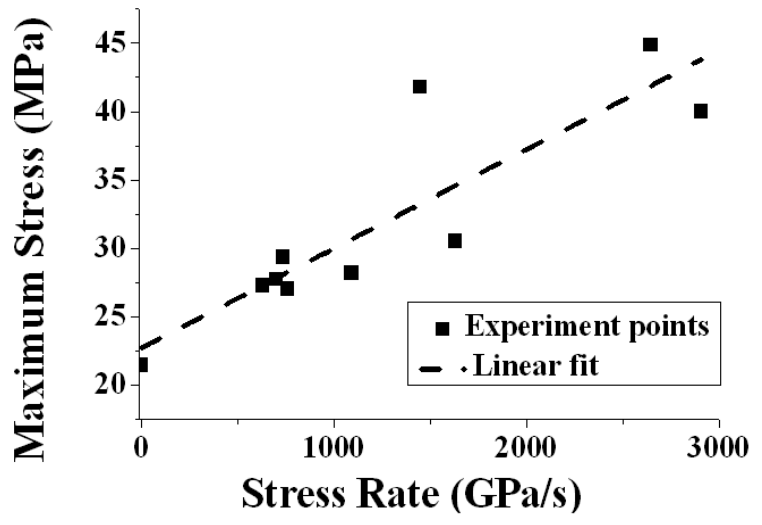

(b)

Figure 2: Test results of CARDIFRC in dynamic splitting [2]. a) Typical stress diagram ( $\sigma^{*}$ is the maximum stress; $t^{*}$ is the fracture time). b) Maximum stress vs stress rate.

For simplicity of understanding, we consider simple test methods, such as the split Hopkinson bar test (the Kolsky method) [10-12] and the spall test [13]. Such tests allow to carry out calculations in one-dimensional statement. For example, Fig. 2 shows the results of dynamic splitting of CARDIFRC (fibre reinforced concrete) [14], which were carried out in the Laboratory of Material Mechanics at the Nizhny Novgorod State University [2].

The initial part of the loading branch (Fig. 2a) corresponds to the growth of the stress and strain. After the stress in the specimen reaches a limiting value, the material begins to fail rapidly accompanied by the formation of micro and macro cracks leading to a significant reduction in the stress with increasing strain.

In spite of the scatter in the data, it is reasonable to conclude that the maximum stress increases with increasing stress rate. The observed time dependence of the maximum stress can be predicted on the basis of the incubation time criterion (1), which in this particular case of fracture takes the form:

$$
\frac{1}{\tau} \int_{t-\tau}^{t} \sigma\left(t^{\prime}\right) d t^{\prime} \leq \sigma_{c}
$$

where $\sigma(t)$ is the time dependence of stress in point of fracture (the average stress in the specimen in case of the SHPB test); $\sigma_{\mathrm{c}}$ is static strength of the material for given type of loading; $\tau$ is the fracture incubation time of the material.

For the well-known formulae from the Strength of Materials we can get a fairly simple relation to calculate the rate dependence of strength. These formulae allow engineers to obtain the values of the material strength, for a given character of loading and specimen geometry, without resorting to lengthy and laborious calculations. In our case, a simple analytical rate dependence of the limit stress can be obtained in the following manner. According to Fig. $2 \mathrm{a}$ an increase in the tensile stress with strain rate can be assumed to be linear until it reaches the maximum value $\sigma^{*}$, so that

$$
\sigma(t)=\dot{\sigma} \cdot t \cdot H(t)=E \cdot \dot{\varepsilon} \cdot t \cdot H(t)
$$

where $\dot{\sigma}$ and $\dot{\varepsilon}$ are the rates of growth of the stress and strain respectively, which we assume to be constant, $E$ is the modulus of elasticity, and $H(t)$ is the Heaviside function. Stress rate determines as the ratio of the maximum stress to the fracture time. We substitute this function into the stress criterion Eq. (2) and find the value of the time to failure $t^{*}$ (using the equality sign) and get 


$$
t^{*}= \begin{cases}\tau / 2+\sigma_{c} / \dot{\sigma}, & t^{*} \geq \tau \\ \sqrt{2 \sigma_{c} \cdot \tau / \dot{\sigma}}, & t^{*}<\tau\end{cases}
$$

which yields the following expression for the limiting stress:

$$
\sigma^{*}=\sigma\left(t^{*}\right)= \begin{cases}\sigma_{c}+\dot{\sigma} \cdot \tau / 2 & t^{*} \geq \tau \\ \sqrt{2 \dot{\sigma} \cdot \sigma_{c} \cdot \tau} & t^{*}<\tau\end{cases}
$$

The incubation time of fracture $\tau$ can be determined by a semi-empirical method. The semi-empirical method consists in fitting of the calculated dependence $\sigma^{*}(\dot{\sigma})$ to the experimental dependence $\sigma^{*}(\dot{\sigma})$ by means of $\tau$ variation. The different algorithms (Gauss-Newton method, the steepest descent method, etc.) can be used to adjust the parameter values in the iterative procedure. The standard way of finding the best fit is to choose the parameters that would minimize the deviations of the theoretical curve(s) from the experimental points. Thereby nonlinear fitting was made to estimate the incubation time values which best describe the data.

It is clear that the experimental values of the studied parameters can deviate from this simple dependence as a result of a scatter. Note that more complex schemes of loading (non-linear loading, three-dimensional problem, etc.) will lead to more complicated analytical expressions. It is more convenient to consider such problems numerically [15].

\section{STRENGTH OF TWO DIFFERENT MATERIALS}

I $\mathrm{n}$ this part, we compare the strength of gabbro-diabase and fibre reinforced concrete (CARDIFRC). Gabbro-diabase is dense, solid, homogeneous rock, characterized by a low degree of resistance to abrasion, frost resistance, and durability. CARDIFRC [14] is an ultra high performance fibre reinforced cement based composite characterised by high compressive and flexural strengths and high toughness. The tests have been performed using the modification of Kolsky method for dynamic splitting (the Brazil test) [16]. Detailed schemes of tests and results were presented in [3] for gabbro-diabase and in [2] for CARDIFRC.

Fig. 3 summarizes the split tests of the fibre reinforced concrete and gabbro-diabase under quasi-static and high strain rates on a semi-logarithmic scale. The curves in Fig. 3 correspond to the calculation by Eq. (2) with the following parameter values: $\sigma_{c}=23 \mathrm{MPa}$ and $\tau=15 \mu \mathrm{s}$ for concrete and $\sigma_{c}=18 \mathrm{MPa}$ and $\tau=70 \mu \mathrm{s}$ for gabbro-diabase.

It is clear from the picture that carrying capacity of both materials increases with the growth of loading rate. However although CARDIFRC has a higher quasi-static split strength than that of gabbro-diabase, its dynamic carrying capacity in splitting is lower at high stress rates $\left(>10^{2.5}\right)$.

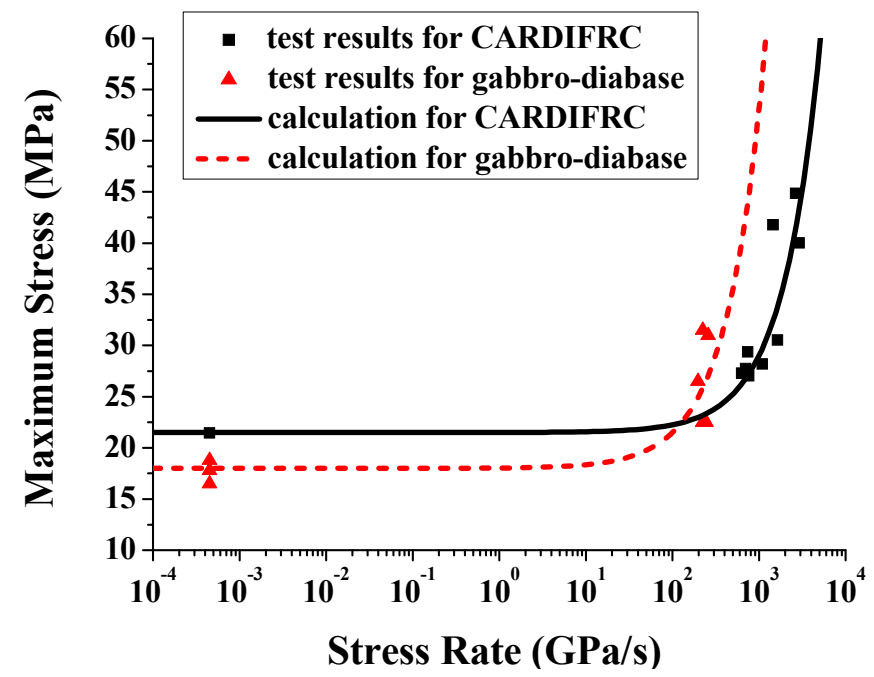

Figure 3: The split tests of CARDIFRC and gabbro-diabase; black squares are the experimental values for CARDIFRC [2]; black line is predictions of Eq. (2) for CARDIFRC $\left(\sigma_{c}=23 \mathrm{MPa}\right.$ and $\left.\tau=15 \mu \mathrm{s}\right)$; red triangles are the experimental values for gabro-diabase [3]; and red dashed is predictions of Eq. (2) for gabbro-diabase $\left(\sigma_{c}=18 \mathrm{MPa}\right.$ and $\left.\tau=70 \mu \mathrm{s}\right)$. 


\section{STRENGTH OF MORTAR AND CONCRETE}

7 he experimental study of the behavior of concrete and mortar at high strain rates in compression were conducted in [4]. Application of techniques of the split Hopkinson bar (SHPB) and plate impact have allowed us to consider a range of loading rates of $10^{2}-10^{4} \mathrm{~s}^{-1}$ with an amplitude of pulses up to $1.5 \mathrm{GPa}$. Both materials have the same processing conditions, and the pure mortar has the same composition as the mortar phase in the concrete. The parameters of the test materials are shown in Tab. 1. The quasi-static compressive strength was determined according to the standard ASTM C39-96. The tests by the Kolsky method were carried out only for mortar.

The results of these experiments are shown in Fig. 4. The curves correspond to the calculation of the maximum stress by the criterion (2). The value of the incubation time $\tau$ can be determined by Eq. (4) (see Table 1). We received the same incubation time for the mortar and concrete. However, despite the lower quasi-static strength compared with that of the mortar, the concrete can show greater strength under dynamic loading (at a given strain rate).

\begin{tabular}{lccccc}
\hline & $\varrho, \mathrm{kg} / \mathrm{m}^{3}$ & $\nu$ & $\boldsymbol{E}, \mathbf{G P a}$ & $\boldsymbol{\sigma}_{c}^{\text {comp }}, \mathbf{M P a}$ & $\tau, \boldsymbol{\mu s}$ \\
Concrete & 2100 & 0.2 & 20 & 46 & 6.5 \\
Pure mortar & 2600 & 0.29 & 45 & 30 & 6.5 \\
\hline
\end{tabular}

Table 1: Parameters of concrete and mortar.

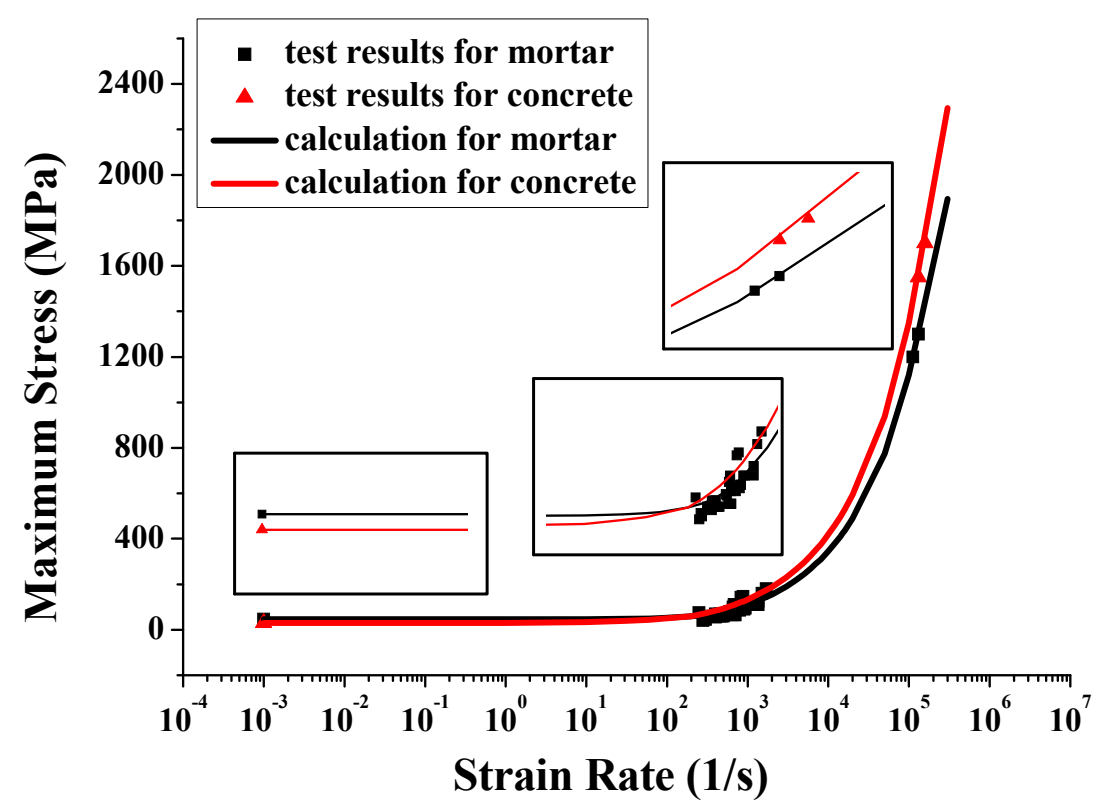

Figure 4: Dependence of limit compression stress on strain rate. Black squares are the experimental values for concrete [4]; black line is predictions of Eq. (2) for concrete; red triangles are the experimental values for mortar [4]; and red line is predictions of Eq. (2) for mortar.

The authors of the experiments [4] explain this effect by existence of hydrostatic pressure in a sample at high speeds of loading that leads to "compression" of microdefects and microcracks in material structure. As concrete can contain many defects (voids, cracks between the cement and the inclusion, etc.), its carrying capacity increases by limiting of the development of defects.

Nevertheless, this effect can be explained with a simple argument, without assumptions about the mechanisms of deformation and fracture. Since the load is linear (see Eq. 3), at high strain rates the limit stress will depend on the modulus of elasticity, that is, the quasi-static strength does not affect on the strength under dynamic loading. Thus, we can set the carrying capacity of concrete by the elasticity of fillers. 


\section{STRENGTH OF DRY AND SATURATED CONCRETE}

I $\mathrm{n}$ the paper [5] the influence of relative humidity of concrete subjected to a large range of stress rate was analyzed and discussed. The Hopkinson Bar Bundle (HBB) was realized to study the high loading rate tensile properties of plain concrete cubes. The experiments were carried out on concrete cured at three different curing conditions (drying at 50 ${ }^{\circ} \mathrm{C} ; 20{ }^{\circ} \mathrm{C}-50 \% \mathrm{RH}$; and saturated) and tested at three strain rates. The results showed that level of free water inside the concrete has an important influence on the carrying capacity of concrete.

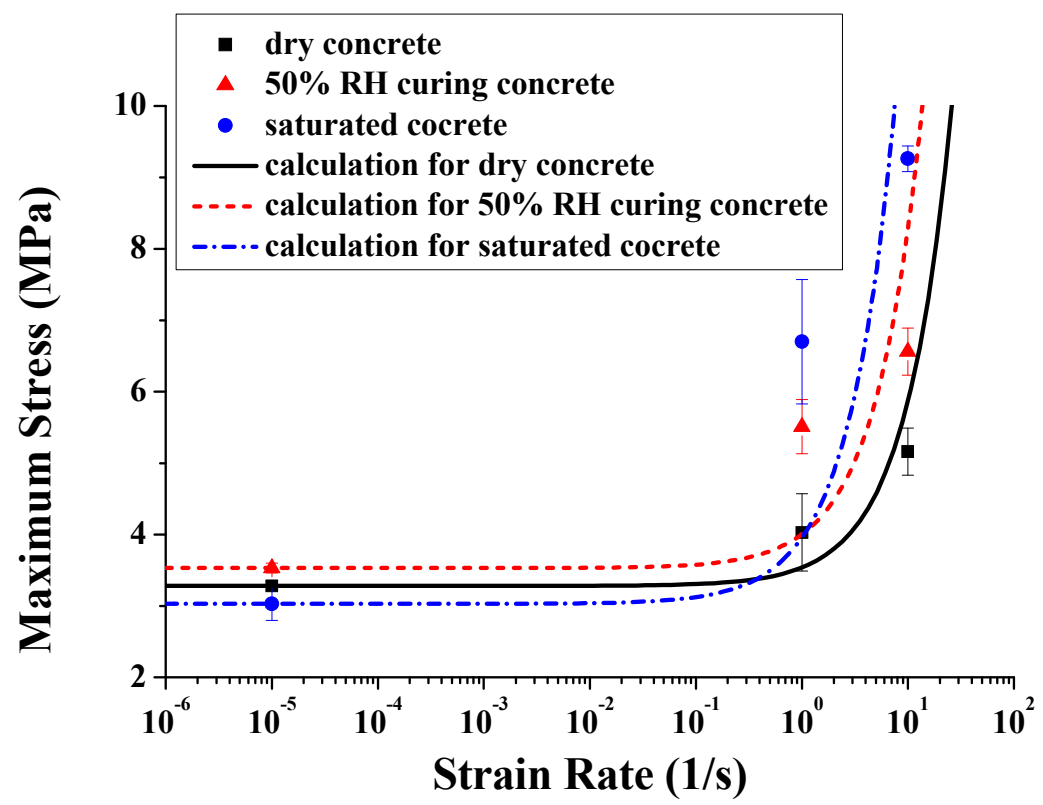

Figure 5: Strain rate effect on maximum tensile stress of concrete with three different curing states. Black squares are the experimental values for dry concrete [5]; black line is predictions of Eq. (2) for dry concrete; red triangles are the experimental values for 50\% $\mathrm{RH}$ curing concrete [5]; and red dash line is predictions of Eq. (2) for 50\% RH curing concrete; blue circles are the experimental values for saturated concrete [5]; blue dash-dot line is predictions of Eq. (2) for saturated concrete.

\begin{tabular}{cccc}
\hline & Dry concrete & $\begin{array}{c}\mathbf{5 0 \%} \mathbf{~ R H} \text { curing } \\
\text { concrete }\end{array}$ & Saturated concrete \\
\hline$\sigma_{\mathrm{c}}, \mathbf{M P a}$ & 3.3 & 3.5 & 3 \\
$\tau, \boldsymbol{\mu s}$ & 12 & 22 & 44 \\
\hline
\end{tabular}

Table 2: Parameters of concrete at different curing conditions.

The results of experiments (points) and the prediction of these results by Eq. (2) (curves) are shown in Fig. 5. The calculations were performed on the basis of the parameters in Table 2. It is clearly seen that the saturated concrete has a lower static strength (limit stress in statics) of the three materials, but one has a higher dynamic strength (the incubation time of fracture). Humidity increases the carrying capacity of concrete at high strain rates and vice versa in statics.

\section{CONCLUSIONS}

I $\mathrm{n}$ this paper, we have analyzed the "effect of substitution" of load-carrying capacity of materials at different strain (stress) rate. The substitution effect means that in spite of the fact that static strength of one material is smaller than that of another one, its dynamic strength measured in terms of incubation time can be essentially higher.

The analysis was conducted based on the incubation time approach, which allows one to separate static strength (limit stress in statics) and dynamic strength (the incubation time of fracture). As the incubation time is a material parameter, 
independent of the loading history, we can estimate and compare load-carrying capacity of materials in a wide range of loading rates.

Three cases of the substitution effect were examined. In the first case, two different materials were compared. It is shown that the fibre reinforced concrete (CARDIFRC) has a lower dynamic strength for a higher static strength compared to the gabbro-diabase.

In the second case, mortar and concrete were considered. It is shown that the substitution effect is also in this case. Moreover the difference between load-carrying capacity of concrete and mortar can depend on the elasticity of aggregate in concrete.

In the third case, concrete with different water saturation was studied. It is shown that saturated concrete has a greater dynamic strength than dry concrete. Thus, humidity can increase the carrying capacity of concrete under dynamic loads, and vice verse under quasi-static loads.

Thus, one of the central problems in testing of dynamic strength properties of rocks and concrete can be associated with measurements of the incubation time parameter. Studies of strain rate (loading rate) phenomenon provide an effective opportunity to examine the incubation stage of the fracture process that is important for predicting critical parameters of external action in a wide range of loading conditions.

\section{REFERENCES}

[1] N.F. Morozov, Y.V. Petrov, Dynamics of fracture. Berlin-Heidelberg-New York: Springer-Velrag (2000).

[2] A.M. Bragov, B.L. Karihaloo, Yu.V. Petrov, A.Yu. Konstantinov, D.A. Lamzin, A.K. Lomunov, I.V. Smirnov, J. of Applied Mechanics and Technical Physics, 53(6) (2012) 926.

[3] A.M. Bragov, A.P. Bolshakov, N.N. Gerdyukov, A.K. Lomunov, S.A. Novikov, I.V. Sergeichev, In: International Conference "V Kharitonov thematic scientific reading" (Sarov, VNIIEF, 2003).

[4] D.L. Grote, S.W. Park, M. Zhou, Int. J. of Impact Eng., 25 (2001) 869.

[5] E. Cadoni, K. Labibes, C. Albertini, M. Berra, M. Giangrasso, Materials and structures, 34 (2001) 21.

[6] Y.V. Petrov, A.A. Utkin, Material Science, 25 (1989) 153.

[7] Y.V. Petrov, N.F. Morozov, ASME J. Appl. Mech., 61 (1994) 710.

[8] Y.V. Petrov, Dokl. Phys., 49 (2004) 246.

[9] Y.V. Petrov, B.L. Karihaloo, V.A. Bratov, A.M. Bragov, Int. J. of Engng. Sci., 61(1) (2012) 3.

[10] A.C. Ross, E.Y. Thompson, J.W. ACI Mater. J., 86(5) (1989) 475.

[11] P.H. Bischoff, S.H. Perry, Mater. Struct., 24 (1991) 425.

[12] F. Akopov, A.M. Bragov, P. Demenko, L. Kruszka, A.K. Lomunov, V. Mineev, L.V. Sergeichev, J. de Physique IV, $110(2003) 225$.

[13] T. Antoun, L. Seaman, D.R. Curran, G.I. Kanel, S.V. Razorenov, A.V. Utkin, Spall Fracture. Springer-Verlag New York (2003).

[14] S.D.P. Benson, B.L. Karihaloo, Magazine Concrete Research, 57 (2005) 347.

[15] V. Bratov, N. Morozov, Y. Petrov. Dynamic Strength of Continuum. St.-Petersburg University Press (2009).

[16] T. Rodriguez, C. Navarro, V. Sanchez-Galvez, Journal de Physique IV, 4(C8) (1994) 101.

[17] A.M. Bragov, B.L. Karihaloo, A.Yu. Konstantinov, D.A. Lamzin, A.K. Lomunov, Bulletin of Nizhny Novgorod University N. Lobachevsky, 4 (2011) 123. (in Russian) 